\title{
Synthesis, Spectroscopic Characterization and Antimicrobial Activity of Some New 2-Substituted Imidazole Derivatives
}

\author{
Asmaa S. Salman, Anhar Abdel-Aziem, Marwa J. S. Alkubbat \\ Department of Chemistry, Faculty of Science, Al-Azhar University, Girls' Branch, Nasr City, Cairo, Egypt \\ Email: salman 2007 ok@yahoo.com
}

Received 15 January 2015; accepted 1 March 2015; published 9 March 2015

Copyright (C) 2015 by authors and Scientific Research Publishing Inc.

This work is licensed under the Creative Commons Attribution International License (CC BY). http://creativecommons.org/licenses/by/4.0/

(c) (i) Open Access

\section{Abstract}

The reaction of imidazole-2-thione derivative 1 with 2 -chloro- $N$ - $p$-tolylacetamide afforded the corresponding 2 -(1H-imidazol-2-ylthio)- $N$ - $p$-tolylacetamide 2 . Reaction compound 2 with different reagents such as $p$-chlorobenzaldehyde and $p$-chlorophenyl diazonium chloride afforded the corresponding arylidene derivative 3 and hydrazone derivative 6 . Reactions of 2 with carbon disulfide in dimethylformamide (DMF) in one equivalent potassium hydroxide afforded intermediate potassium sulphide salt 8, which treatment with dilute hydrochloric acid and phenacyl bromide afforded the corresponding 2-[p-tolylcarbamoyl]ethanedithioic acid 9 and 3-[benzo-ylmethylthio]- $N$ - $p$-tolyl-3-thioxo-propaneamide 10 . While the reaction 2 with carbon disulphide in the presence of two equivalent potassium hydroxide in DMF gave non-isolated potassium salt 11, which was allowed to react with halogenated compounds namely ethyl chloroacetate and methyl iodide afforded the corresponding 3, 3-bis[(ethoxycarbonyl)methylthio]- $N$ - $p$-tolylacrylamide 12 and 3,3bis-(methylthio)- $N$ - $p$-tolylacrylamide 13 respectively. Reaction 2 with phenyl isothiocyanate in basic DMF yielded the intermediate potassium sulphide salt 18. Acidification 18 with dilute hydrochloric acid afforded the corresponding thiocarbamoyl derivative 19. Treatment of intermediate 18 with methyl iodide, phenacyl bromide and ethylchloroacetate afforded the 3-anilino-3-(methylthio)- $N$ - $p$-tolylacrylamide 20, 2-(1,3-thiazol-2(3H)-ylidene)- $N$ - $p$-tolylacetamide 21 and 2-(4-oxo-3phenyl-1,3-thiazolidin-2-ylidene)- $N$ - $p$-tolylacetamide 22 respectively. The structure of the newly synthesized compounds has been confirmed by elemental analysis and spectra data. Synthesized compounds $2,3,6,13,15 a, 15 b, 17,20,21,22$ and 23 were screened for their antibacterial activities in vitro against Gram-positive (Staphylococcus aureus and Bacillus subtilis), Gram-negative (Pseudomonas aeuroginosa and Escherichia coli) and antifungal activities against (Aspergillus fumigates, Syncephalastrum racemosum, Geotrichum candidum and Candida albicans). 
Keywords

Imidazole-2-Thione, $\alpha$-Oxoketen Dithioacetals, Thiocarbamyl, Thiazole, Antibacterial, Antifungal

\section{Introduction}

Aromatic heterocycles are valuable synthetic templates for the preparation of new compounds with specific biological, pharmaceutical and material properties. The pursuit of these properties requires efficient synthetic routes that allow rapid construction of diverse aromatic heterocycles with defined substitution patterns. Therefore, $\alpha-$ oxoketen dithioacetals and thiocarbamyl as the organic synthetic intermediates have been widely used in the formation of alicyclic, aromatic and heterocyclic compounds [1]-[4]. In view of the above and in continuation of our studies on the synthesis of heterocyclic compounds exhibiting biological activity, we report here the synthesis of some novel heterocycles compounds incorporating imidazole moiety from $\alpha$-oxoketen dithioacetals and thiocarbamyl.

\section{Material and Methods}

\subsection{Experiment}

All melting points were determined in open glass capillaries on a Gallen kamp apparatus and are uncorrected. IR spectra $\left(\mathrm{cm}^{-1}\right)$ were recorded on a Pye-Unicam spectrophotometer type 1200 using $\mathrm{KBr}$ discs. $1 \mathrm{H}$-NMR spectra were recorded on a Varian EM-390 (90 MHz) spectrometer using TMS as an internal standard and dimethyl sulphoxide (DMSO-d6) as a solvent. Chemical shifts were expressed in $\delta(\mathrm{ppm})$ values and mass spectra were determined on Finnigan Incos 500 (70 ev). Elemental analyses were determined using a Parkin-Elmer 240C Microanalyzer. The microanalyses were performed at the Microanalytical Unit, Faculty of Science, Cairo University.

\subsubsection{2-[1-(4-Chlorophenyl)-4,5-diphenyl- $1 H$-imidazol-2-ylthio]- $N$ - $p$-tolylacetamide 2}

A mixture of $1(0.01 \mathrm{~mol})$ and 2-chloro- $N$-p-tolylacetamide $(0.01 \mathrm{~mol})$ in DMF (25 ml) contain few drop of triethylamine was heated under reflux for $6 \mathrm{~h}$. The reaction mixture was left to cool and then poured to ice cooled water $(100 \mathrm{ml})$. The solid product that formed was filtered off, dried well and recrystallized from ethanol to give 2 as pall yellow crystals. Yield: $70 \%$. M.p.: $156^{\circ} \mathrm{C}-158^{\circ} \mathrm{C}$; IR (K Br) $\mathrm{cm}^{-1}: 3246(\mathrm{NH}), 1683(\mathrm{C}=\mathrm{O}), 3041$, $2931(\mathrm{CH}), 1600(\mathrm{C}=\mathrm{N})$; $1 \mathrm{H}-\mathrm{NMR}$ (DMSO-d6) $\delta \mathrm{ppm}: 4.11$ (s, 2H, $\left.\mathrm{CH}_{2}\right), 2.24\left(\mathrm{~s}, 3 \mathrm{H}, \mathrm{CH}_{3}\right), 10.36(\mathrm{~s}, 1 \mathrm{H}, \mathrm{NH})$, 7.09 - 7.47 (m, 18H, Ar-H); MS m/z (\%): 510 (M+2, 26.25), 404 (17.60), 375 (27.06), 362 (8.612), 193 (100), 147 (10.5); Anal. Calcd. for $\mathrm{C}_{30} \mathrm{H}_{24} \mathrm{ClN}_{3} \mathrm{OS}$ (510.05): C, 70.64; H, 4.74; Cl, 6.95; N, 8.24; S, 6.29. Found: C, 70.44; H, 4.54; Cl, 6.65; N, 8.04; S, 6.09.

\subsubsection{2-[1-(4-Chlorophenyl)-4,5-diphenyl-1H-imidazol-2-ylthio]-3-(4-chlorophenyl)- $N$ - $p$ - tolylacrylamide 3}

A mixture of $2(0.01 \mathrm{~mol})$ and $p$-chlorobenzaldehyde $(0.01 \mathrm{~mol})$ in ethanol $(30 \mathrm{ml})$ containing few drop of piperidine $(0.5 \mathrm{ml})$ was refluxed for $3 \mathrm{~h}$. The reaction mixture was left to cool then poured onto ice water contain few drops of $\mathrm{HCl}$ and the obtained solid was recrystallized from ethanol to give 3 as white crystals. Yield: $60 \%$. M.p.: $170^{\circ} \mathrm{C}-171^{\circ} \mathrm{C}$; IR (KBr) cm ${ }^{-1}: 3247(\mathrm{NH}), 1687(\mathrm{C}=\mathrm{O}), 3046,2951(\mathrm{CH}) ; 1 \mathrm{H}-\mathrm{NMR}$ (DMSO-d6) $\delta$ ppm: 2.24 (s, 3H, $\left.\mathrm{CH}_{3}\right), 10.31(\mathrm{~s}, 1 \mathrm{H}, \mathrm{NH}), 7.09-7.47$ (m, 23H, Ar-H and =CH); MS m/z (\%): $632\left(\mathrm{M}^{+}, 11.24\right), 548$ (23.22), 520 (398.33), 430 (23.22), 386 (27.34), 309 (24.34), 80 (100); Anal. Calcd. for $\mathrm{C}_{37} \mathrm{H}_{27} \mathrm{Cl}_{2} \mathrm{~N}_{3} \mathrm{OS}$ (632.60): C, 70.25; H, 4.30; Cl, 11.21; N, 6.64; S, 5.07. Found: C, 70.00; H, 4.00; Cl, 11.00; N, 6.44; S, 5.00.

\subsubsection{5-(4-Chlorophenyl)-4-[1-(4-chlorophenyl)-4,5-diphenyl-1H-imidazol-2-ylthio]- $N$ - $p$-tolyl- $1 \mathrm{H}$-pyrazole-3-amine 4}

A mixture of $3(0.01 \mathrm{~mol})$ and hydrazine hydrate $(0.01 \mathrm{~mol})$ in $30 \mathrm{ml}$ absolute ethanol was added few drops of glacial acetic and refluxed for 8 - $10 \mathrm{~h}$. After completion of the reaction, excess of solvent was distilled off; the separated solid was filtered, washed with water, and recrystallized from methanol to give $\mathbf{4}$ as yellow crystals. 
Yield: 55\%. M.p.:138 ${ }^{\circ} \mathrm{C}-140^{\circ} \mathrm{C}$; IR (KBr) cm ${ }^{-1}$ : 2922, 3054 (CH), 3437 (NH); 1H-NMR (DMSO-d6) $\delta$ ppm: 1.74 (s, 3H, $\mathrm{CH}_{3}$ ), $7.17-7.49$ (m, 23H, Ar-H and NH), 9.40 (s, 1H, NH); MS m/z (\%): $644\left(\mathrm{M}^{+}, 11.02\right), 630$ (12.44), 558 (9.13), 537 (5.98), 511 (8.03), 483 (6.61), 464 (12.76), 405 (17.64), 333 (9.29), 388 (9.49), 233 (100); Anal. Calcd. for $\mathrm{C}_{37} \mathrm{H}_{27} \mathrm{Cl}_{2} \mathrm{~N}_{5} \mathrm{~S}$ (644.61): C, 68.94; H, 4.22; Cl, 11.00; N, 10.86; S, 4.97. Found: C, 68.64; H, 4.00; Cl, 10.99; N, 10.66; S, 4.67.

\subsubsection{5-(4-Chlorophenyl)-4-[1-(4-chlorophenyl)-4,5-diphenyl-1H-imidazol-2-ythio]-1-phenyl- $N$ - p-tolyl-1H-pyrazol-3-amine 5}

A mixture of $3(0.01 \mathrm{~mol})$, phenyl hydrazine $(0.01 \mathrm{~mol})$, glacial acetic acid $(20 \mathrm{ml})$ and few drop of $\mathrm{HCl}$ was refluxed for 8 - $10 \mathrm{~h}$. The reaction mixture was then left to cool at room temperature, and poured onto ice cold water $(100 \mathrm{ml})$. The solid product was collected by filtration and recrystallized from acetic acid to give $\mathbf{5}$ as grey crystals. Yield: $60 \%$. M.p.: $290^{\circ} \mathrm{C}-292^{\circ} \mathrm{C}$; IR (KBr) cm ${ }^{-1}: 3426(\mathrm{NH}), 3048,2926(\mathrm{CH}), 1594(\mathrm{C}=\mathrm{N})$; $1 \mathrm{H}-\mathrm{NMR}$ (DMSO- d6) $\delta$ ppm: 1.90 (s, 3H, $\mathrm{CH}_{3}$ ), 7.13 - 7.48 (m, 27H, Ar-H), 13.03 (s, $1 \mathrm{H}, \mathrm{NH}$ ); MS m/z (\%): 720 (M+, 8.26), 628 (7.05), 554 (6.68), 538 (7.90), 615 (8.51), 512 (8.26), 423 (6.93), 266 (11.42); Anal. Calcd. for $\mathrm{C}_{43}$ $\mathrm{H}_{31} \mathrm{Cl}_{2} \mathrm{~N}_{5} \mathrm{~S}$ (720.71):C, 71.66; H, 4.34; Cl, 9.84; N, 9.72; S, 4.45. Found: C, 71.36; H, 4.04; Cl, 9.54; N, 9.42; S, 4.25.

\subsubsection{2-[2-(4-Chlorophenyl)hydrazono]-2-[1-(4-chlorophenyl)-4,5-diphenyl-1H-imidazol-2- ylthio]- $N$ - $p$-tolylacetamide 6}

To a cold solution of $2(0.01 \mathrm{~mol})$ in pyridine $(30 \mathrm{ml})$ was added with continuous stirring 4-chloro phenyl-diazonium salt $(0.01 \mathrm{~mol})$ [prepared by adding sodium nitrite $(0.02 \mathrm{~mol})$ in water $(8 \mathrm{ml})$ to a cold solution of the $p$ chloroaniline in the appropriate amount of hydrochloric acid]. The reaction mixture was stirred at room temperature for $2 \mathrm{~h}$, and the solid products, so formed, were collected by filtration and recrystallized from benzene to give 6 as red crystals. Yield: 67\%. M.p.: $118^{\circ} \mathrm{C}-120^{\circ} \mathrm{C}$; IR $\left(\mathrm{KBr}, \mathrm{cm}^{-1}\right)$ : 3259, $3136(\mathrm{NH}), 1604(\mathrm{C}=\mathrm{N}), 1686$ (C=O); 1H-NMR ((DMSO-d6) $\delta$ ppm: 2.24 (s, 3H, $\left.\mathrm{CH}_{3}\right), 7.09$ - 7.47 (m, 22H, Ar-H), 8.58(s, 1H, NH), 10.32(s, $1 \mathrm{H}, \mathrm{NH}) ; \mathrm{MS} \mathrm{m} / \mathrm{z}$ (\%): $648\left(\mathrm{M}^{+}, 40.12\right), 543(43.83), 471(45.68), 442$ (33.33), 362 (33.95), 367 (33.95), 287 (55.02), 211 (35.80), 196 (54.94), 77 (100); Anal. Calcd. for: $\mathrm{C}_{36} \mathrm{H}_{27} \mathrm{Cl}_{2} \mathrm{~N}_{5} \mathrm{OS}(648.60)$ ) C, 66.66; $\mathrm{H}, 4.20$; $\mathrm{Cl}$, 10.93; N, 10.80; S, 4.94. Found: C, 66.36; H, 4.0 0; Cl, 10.73; N 10.50; S, 4.74.

\subsubsection{2-[1-(4-Chlorophenyl)-4,5-diphenyl-1H-imidazol-2-ylthio]-2-[p-tolylcarbamoyl] ethanedithioicacid 9}

To suspension of finely powdered potassium hydroxide $(0.01 \mathrm{~mol})$ in dry DMF $(20 \mathrm{ml})$ at $0^{\circ} \mathrm{C}$, the acetamide derivative $2(0.01 \mathrm{~mol})$ was added, the resulted mixture was cooled at $10^{\circ} \mathrm{C}$ in an ice bath, then carbon disulfide $(0.01 \mathrm{~mol})$ was added slowly over the course of $10 \mathrm{~min}$. After complete addition, stirring of the reaction mixture was continued for $6 \mathrm{~h}$. Then hydrochloric acid ( $2 \mathrm{M}, 20 \mathrm{ml})$ was added drop wise and stirring continued for additional $1 \mathrm{~h}$. Then, the reaction mixture was poured into ice water. The solid product that formed was filtered off, dried, and recrystallized from the ethanol to give 9 as yellow crystals. Yield: $75 \%$. M.p.: $222^{\circ} \mathrm{C}-224^{\circ} \mathrm{C}$; IR (KBr, cm ${ }^{-1}$ : $3231(\mathrm{NH}), 1682$ (CO), 3043, $2855(\mathrm{CH}), 1271$ (C=S); MS m/z (\%): 587 (M+1, 4.08), $586\left(\mathrm{M}^{+}\right.$, 4.53), 553 (3.18), 520 (5.47), 480 (3.35), 415 (3.46), 233 (100), 221 (3.80), 77 (96.65); Anal. Calcd. for $\mathrm{C}_{31} \mathrm{H}_{24} \mathrm{ClN}_{3} \mathrm{OS}_{3}$ (586.19): C, 63.52; H, 4.13; Cl, 6.05; N, 7.17; S, 16.41. Found: C, 63.32; H, 4.00; Cl, 6.00; N, 7.00; S, 16.21 .

\subsubsection{3-[Benzoylmethylthio]-2-[1-(4-chlorophenyl)-4,5-diphenyl-1H-imidazol-2-ylthio]- $N$ - $p$ - tolyl-3-thioxo-propaneamide 10}

To suspension of finely powdered potassium hydroxide $(0.01 \mathrm{~mol})$ in dry DMF $(20 \mathrm{ml})$ at $0^{\circ} \mathrm{C}$, the acetamide derivative 2 ( $0.01 \mathrm{~mol}$ ) was added, the resulted mixture was cooled at $10^{\circ} \mathrm{C}$ in an ice bath, then carbon disulfide ( $0.01 \mathrm{~mol})$ was added slowly over the course of $10 \mathrm{~min}$. After complete addition, stirring of the reaction mixture was continued for $6 \mathrm{~h}$. Then cooled again to $0^{\circ} \mathrm{C}$, phenacyl bromide $(0.01 \mathrm{~mol})$ was added slowly over the course of $10 \mathrm{~min}$. After complete addition, stirring of the reaction mixture was continued for $6 \mathrm{~h}$. Then poured into crushed ice, the resulting precipitate was filtrated off, dried and recrystallized from ethanol to give $\mathbf{1 0}$ as yellow crystals. Yield: $80 \%$. M.p.: $146^{\circ} \mathrm{C}-148^{\circ} \mathrm{C}$; IR $\left(\mathrm{KBr}, \mathrm{cm}^{-1}\right)$ : $3416(\mathrm{NH}), 1762,1686(\mathrm{CO}), 1280(\mathrm{C}=\mathrm{S})$, $1600(\mathrm{C}=\mathrm{N}), 3052$, 2967, 2913 (CH); 1H-NMR (DMSO-d6) $\delta$ ppm: 2.25 (s, 3H, $\left.\mathrm{CH}_{3}\right), 4.39\left(\mathrm{~s}, 2 \mathrm{H}, \mathrm{CH}_{2}\right.$ ), 4.57 (s, H, CH), 7.10 - 8.02 (m, 23H, Ar-H), 10.31 (s, 1H, NH); Anal. Calcd. For $\mathrm{C}_{39} \mathrm{H}_{30} \mathrm{ClN}_{3} \mathrm{O}_{2} \mathrm{~S}_{3}$ (704.32): C, 66.51; 
H, 4.29; Cl, 5.03; N, 5.97; S, 13.66. Found: C, 66.31; H, 4.09; Cl, 5.00; N, 5.77; S, 13.46.

\subsubsection{3,3-Bis[(ethoxycarbonyl)methylthio]-2-[1-(4-chlorophenyl)-4,5-diphenyl-1H-imidazol-2- ylthio]- $N$ - $p$-tolylacrylamide 12}

To suspension of finely powdered of potassium hydroxide $(0.02 \mathrm{~mol})$ in dry $\operatorname{DMF}(20 \mathrm{ml})$ at $0^{\circ} \mathrm{C}$ the acetamide derivative $2(0.01 \mathrm{~mol})$ was added, the resulted mixture was cooled at $10^{\circ} \mathrm{C}$ in an ice bath, then carbon disulfide $(0.01 \mathrm{~mol})$ was added slowly over the course of $10 \mathrm{~min}$. After complete addition, stirring of the reaction mixture was continued for $6 \mathrm{~h}$. Then cooled again to $0^{\circ} \mathrm{C}$, ethyl chloroacetate $(0.02 \mathrm{~mol})$ was added slowly over the course of $10 \mathrm{~min}$. After complete addition, stirring of the reaction mixture was continued for $6 \mathrm{~h}$. Then poured into crushed ice, the resulting precipitate was filtrated off, dried and recrystallized from benzene to give $\mathbf{1 2}$ as yellow crystals. Yield: $65 \%$. M.p.: $120^{\circ} \mathrm{C}-122^{\circ} \mathrm{C}$; IR $\left(\mathrm{KBr}, \mathrm{cm}^{-1}\right)$ : $3252(\mathrm{NH}), 1736,1685$ (CO), 3049, 2980, 2925 (CH); 1H-NMR (DMSO-d6) $\delta$ ppm: $1.13-1.23$ (m, 6H, 2- $\left.\mathrm{CH}_{2}-\mathrm{CH}_{3}\right), 4.33$ (s, 2H, $\left.\mathrm{CH}_{2}\right), 4.37$ (s, 2H, $\mathrm{CH}_{2}$ ), 4.04 - 4.153 (m, $4 \mathrm{H}, 2 \mathrm{CH}_{2}-\mathrm{CH}_{3}$ ), 2.24 (s, 3H, $\left.\mathrm{CH}_{3}\right), 7.09$ - 7.51 (m, 18H, Ar-H), 10.32 (s, $\left.1 \mathrm{H}, \mathrm{NH}\right)$; Anal. Calcd. for $\mathrm{C}_{39} \mathrm{H}_{36} \mathrm{ClN}_{3} \mathrm{O}_{5} \mathrm{~S}_{3}$ (758.37): C, 61.77; H, 4.78; Cl, 4.67; N, 5.54; S, 12.68. Found:C, 61.67; H, 4.58; Cl, 4.47; N, 5.34; S, 12.38.

\subsubsection{3,3-Bis(methylthio)-2-[1-(4-chlorophenyl)-4,5-diphenyl-1H-imidazol-2-ylthio]- $N$ - $p$ - tolylacrylamide 13}

Compound 13 was synthesized as mentioned above in synthesis of 12 but using methyl iodide (0.02 mol) instead of ethyl chloroacetate, the resulting product was recrystallized from ethanol to give $\mathbf{1 3}$ as yellow crystals. Yield: 60\%. M.p.: $166^{\circ} \mathrm{C}-168^{\circ} \mathrm{C}$; IR (KBr, $\left.\mathrm{cm}^{-1}\right)$ : $3438(\mathrm{NH}), 1674(\mathrm{CO}), 3050,2919$ (CH); 1H-NMR (DMSO-d6) $\delta$ ppm 2.21 (s, 3H, $\mathrm{CH}_{3}$ ), $7.18-7.68$ (m, 19H, Ar-H and NH ), 2.28 (s, 6H, 2SCH${ }_{3}$ ); MS m/z (\%): 614 ( $\mathrm{M}^{+}$, 20.64), 611(16.71), 568 (13.154), 506 (19.41), 415 (13.27), 315 (14.74), 252 (15.48), 206 (17.44), 237 (15.97), 75 (100); Anal. Calcd. for $\mathrm{C}_{33} \mathrm{H}_{28} \mathrm{ClN}_{3} \mathrm{OS}_{3}(614.24)$ : C, 64.53; H, 4.59; Cl, 5.77; N, 6.84; S, 15.66 . Found: C, 64.23; H, 4.39; Cl, 5.57; N, 6.64; S, 15.36.

\subsubsection{2-[1-(4-Chlorophenyl)-4,5-diphenyl-1H-imidazol-2-ylthio]-3,3-dihydrazino- $N$ - $p$ - tolylacrylamide 14}

A mixture of compound $13(0.01 \mathrm{~mol})$ and hydrazine hydrate $(80 \%, 0.02 \mathrm{~mol})$ was heated under reflux for $4 \mathrm{~h}$, then left to cool. The obtained solid product was triturated with ethanol $(10 \mathrm{ml})$, filtered off, washed with ethanol, dried and recrystallized from butanol afford compound 14 as white crystals. Yield: $60 \%$. M.p.: $210^{\circ} \mathrm{C}$ $213^{\circ} \mathrm{C}$; IR(KBr, cm $\left.{ }^{-1}\right)$ : 3297, 3269, 3196, $3126\left(\mathrm{NH}_{2}, \mathrm{NH}\right), 1682$ (CO), 3061, 2959 (CH); 1H-NMR (DMSO-d6) $\delta$ ppm: 2.24 (s, 3H, $\left.\mathrm{CH}_{3}\right), 7.09-7.56$ (m, 19H, Ar-H and NH), 10.33 (s, 1H, NH ), $10.51(\mathrm{~s} .1 \mathrm{H}, \mathrm{NH}), 4.84$ (s,

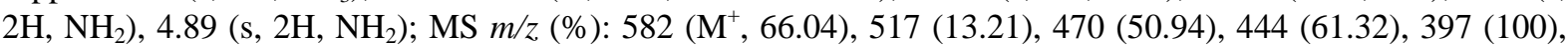
381 (58.49), 321 (24.53); Anal. Calcd. for $\mathrm{C}_{31} \mathrm{H}_{28} \mathrm{ClN}_{7} \mathrm{OS}$ (582.11): C, 63.96; H, 4.85; Cl, 6.09; N, 16.84; S, 5.51. Found: C, 63.66; H, 4.65; Cl, 6.00; N, 16.64; S, 5.41.

\subsubsection{Reaction of 3,3-Bis(methylthio)-2-[1-(4-chlorophenyl)-4,5-diphenyl-1H-imidazol-2- ylthio]- $N$ - $p$-tolylacrylamide 13 with Amines}

A mixture of $13(0.01 \mathrm{~mol})$ suitable amine such as $o$-phenylenediamine, $o$-aminophenol and/or p-chloroaniline $(0.01 \mathrm{~mol})$ in DMF $(25 \mathrm{ml})$ was heated under reflux for $6 \mathrm{~h}$. The reaction mixture was left to cool and then poured to ice cooled water $(100 \mathrm{ml})$. The solid product that formed was filtered off, dried well and recrystallized from appropriate solvent.

2-(1H-Benzimidazol-2-yl)-2-[1-(4-chlorophenyl)-4,5-diphenyl-1H-imidazol-2-ylthio]- $N$-p-tolylacetamide 15a

White powder. Yield: $65 \%$. M.p.: $238^{\circ} \mathrm{C}-240^{\circ} \mathrm{C}$ (ethanol and DMF); $\mathrm{IR}\left(\mathrm{KBr}, \mathrm{cm}^{-1}\right)$ : 3245, 3188 (NH); 3055, 2924, 2865 (CH), 1659 (CO), 1607 (C=N); 1H-NMR (DMSO-d6) $\delta$ ppm: 2.25 (s, 3H, $\mathrm{CH}_{3}$ ), 7.09 - 7.53 (m, 22H, Ar-H); 7.95 (s, 1H, NH), 10.30 (s, 1H, NH), 4.61(s, 1H, CH); MS m/z (\%): 626 (M+2, 29.10), 491 (212.31), 424 (29.10), 391 (31.97), 379 (35.25), 328 (26.64), 75 (100); Anal. Calcd. for $\mathrm{C}_{37} \mathrm{H}_{28} \mathrm{ClN}_{5} \mathrm{OS}$ (626.17): C, 70.97; H, 4.51; Cl, 5.66; N, 11.18; S, 5.12. Found: C, 70.67; H, 4.21; Cl, 5.46; N, 11.08; S, 5.02.

\section{2-(1,3-Benzoxazol-2-yl)-2-[1-(4-chlorophenyl)-4,5-diphenyl-1 $H$-imidazol-2-ylthio]- $N$-p-tolylacetamide 15b}

Pale grey crystals. Yield: 55\%. M.p.: $176^{\circ} \mathrm{C}-178^{\circ} \mathrm{C}$ (ethanol); IR (KBr, cm ${ }^{-1}$ ) 3246 (NH); 1687 (CO), 1600 
(C=N), 3048, 2922, 2864 (CH); 1H-NMR (DMSO-d6) $\delta$ ppm: 2.24 (s, 3H, $\mathrm{CH}_{3}$ ), 7.09 - 7.47 (m, 22H, Ar-H); 10.43 (s, 1H, NH), 4.16 (s, 1H, CH); MS m/z (\%): 627 (M $\left.\mathrm{M}^{+}, 11.96\right), 611$ (11.52), 516 (10.49), 492 (8.57), 405 (8.86), 330 (9.16), 264 (10.34), 173 (10.49), 77 (100); Anal. Calcd. for $\mathrm{C}_{37} \mathrm{H}_{27} \mathrm{Cl} \mathrm{N}_{4} \mathrm{O}_{2} \mathrm{~S}$ ( 627.15):C, 70.86; H, 4.34; Cl, 5.65; N, 8.93; S, 5.11. Found: C, 70.66; H, 4.04; Cl, 5.45; N, 8.73; S, 5.00.

2-[1-(4-Chlorophenyl)-4,5-diphenyl-1 $\mathrm{H}$-imidazol-2-ylthio]-3-(4-chlorophenylamino]-3-(methylthio)$N$-p-tolylacrylamide 16

White powder. Yield: 65\%. M.p.: $190^{\circ} \mathrm{C}-192^{\circ} \mathrm{C}$ (ethanol); IR (KBr, $\mathrm{cm}^{-1}$ ) 3246, 3191 (NH); 1687 (CO), 1599 (C=N); 1H-NMR (DMSO-d6) $\delta$ ppm: 2.25 (s, 3H, $\mathrm{CH}_{3}$ ), 2.39 (s, 3H, $\left.\mathrm{SCH}_{3}\right), 7.09-7.62$ (m, 23H, Ar-H and NH); 10.29 (brs, 1H, NH); MS m/z (\%): 693 (M+1 53.29), 631 (43.13) 622 (40.72), 607 (40.72), 471 (35.93), 330 (53.89), 234 (35.93), 257 (51.51), 272 (34.13), 77 (100); Anal. Calcd. for $\mathrm{C}_{38} \mathrm{H}_{30} \mathrm{Cl}_{2} \mathrm{~N}_{4} \mathrm{OS}_{2}$ (693.70): C, 65.79; H, 4.36; Cl, 10.22; N, 8.08; S, 9.24. Found: C, 65.59; H, 4.26; Cl, 10.02; N, 8.00; S, 9.04.

\subsubsection{4-[1-(4-Chlorophenyl)-4,5-diphenyl-1H-imidazol-2-ylthio]-3-( $p$-tolylamino)-5-} (methylthio)-1H-pyrrole-2-carboxylic Acid 17

A mixture of compound $13(0.01 \mathrm{~mol})$ and glycine $(0.01 \mathrm{~mol})$ in ethanol $(30 \mathrm{ml})$ containing triethylamine $(5$ drops) was heated under reflux for $8 \mathrm{~h}$. The formed solid product was filtered off, dried and recrystallized from ethanol to give 17 as a yellow powder. Yield: $62 \%$. M.p.: $180^{\circ} \mathrm{C}-182^{\circ} \mathrm{C}$; $\mathrm{IR}\left(\mathrm{KBr}, \mathrm{cm}^{-1}\right)$ : $3436(\mathrm{OH}), 3247$, $3184(\mathrm{NH}) ; 1773(\mathrm{CO}), 1607$ (C=N); 1HNMR ((DMSO-d6) $\delta$ ppm: 2.25 (s, 3H, $\left.\mathrm{CH}_{3}\right), 2.29$ (s, 3H, $\left.\mathrm{SCH}_{3}\right), 7.09$ - 7.47 (m, 20H, Ar-H and 2NH); 13.29 (s, 1H, OH); MS m/z (\%): 623 (M+, 7.89 ), 579 (13.92), 575 (21.87), 516 (10.93), 480 (19.28), 470 (14.12), 390 (12.33), 293 (15.52), 261 (13.12), 214 (24.25), 77 (100); Anal. Calcd. for $\mathrm{C}_{34} \mathrm{H}_{27} \mathrm{ClN}_{4} \mathrm{O}_{2} \mathrm{~S}_{2}$ (623.18): C, 65.53; H, 4.37; Cl, 5.69; N, 8.99; S, 10.29. Found: C, 65.23; H, 4.17; Cl, 5.49; N, 8.79; S, 10.09 .

\subsubsection{3-Anilino-2-[1-(4-chlorophenyl)-4,5-diphenyl-1H-imidazol-2-ylthio]- $N$ - $p$-tolyl-3- thioxopropan 19}

To a stirred solution of powdered potassium hydroxide $(0.02 \mathrm{~mol})$ in DMF $(20 \mathrm{ml})$, compound 2 (0.02 mol) was added. After stirring for $30 \mathrm{~min}$, phenyl isothiocyanate $(0.02 \mathrm{~mol})$ was added to the resulting mixture, stirring was continued for $6 \mathrm{~h}$, and then poured over crushed ice containing hydrochloric acid. The solid product that formed was filtered off, washed with water, dried and recrystallized from ethanol to give $\mathbf{1 9}$ as yellow crystals. Yield: 64\%. M.p.: $186^{\circ} \mathrm{C}-188^{\circ} \mathrm{C}$; IR $\left(\mathrm{KBr}, \mathrm{cm}^{-1}\right)$ : 3246, $3191(\mathrm{NH}), 3084,2925(\mathrm{CH}), 1656(\mathrm{C}=\mathrm{O}), 1606$ $(\mathrm{C}=\mathrm{N}), 1238$ (C=S); 1H-NMR(DMSO-d6) $\delta$ ppm: 2.24 (s, 3H, $\left.\mathrm{CH}_{3}\right), 10.40$ (s, 1H, NH), 10.20 (s, 1H, NH), 7.05 - 7.59 (m, 23H, Ar-H), 4.12 (s, 1H, CH); MS m/z (\%): 645 (M+2 23), 629 (22.03), 612 (23), 554 (18.32), 521 (20.91), 512 (23), 526 (18.12), 316 (22.65), 300 (187.12), 224 (29.21), 192 (20.21), 80 (100); Anal. Calcd. for $\mathrm{C}_{37} \mathrm{H}_{29} \mathrm{ClN}_{4} \mathrm{OS}_{2}$ (645.23): C, 68.87; H, 4.53; Cl, 5.49; N, 8.68; S, 9.94. Found: C, 68.67; H, 4.23; Cl, 5.29 N, 8.48; S, 9.64

\subsubsection{3-Anilino-2-[1-(4-chlorophenyl)-4,5-diphenyl-1 $H$-imidazol-2-ylthio]-3-(methylthio)- $N$ - $p$ - tolylacrylamide 20}

To a stirred solution of potassium hydroxide $(0.01 \mathrm{~mol})$ in DMF $(20 \mathrm{ml})$ was added compound $2(0.01 \mathrm{~mol})$. After the mixture was stirred for $30 \mathrm{~min}$, phenyl isothiocyanate $(0.01 \mathrm{~mol})$ was added to the resulting mixture. Stirring was continued for $6 \mathrm{~h}$, and then methyl iodide $(0.01 \mathrm{~mol})$ was added and stirring was continued for $6 \mathrm{~h}$. The reaction mixture was poured onto ice-cold water. The solid product that formed was collected by filtration, dried and recrystallized from ethanol to give compound 20 as yellow crystals. Yield: $62 \%$. M.p.: $234^{\circ} \mathrm{C}-236^{\circ} \mathrm{C}$; IR $\left(\mathrm{KBr}, \mathrm{cm}^{-1}\right)$ : 3246, $3191(\mathrm{NH}), 1656(\mathrm{C}=\mathrm{O}), 1604(\mathrm{C}=\mathrm{N}), 3086,2874(\mathrm{CH})$; 1H-NMR (DMSO-d6) $\delta$ ppm: 2.25 (s, $\left.3 \mathrm{H}, \mathrm{CH}_{3}\right), 2.54$ (s, 3H, $\left.\mathrm{SCH}_{3}\right), 10.30(\mathrm{~s}, 1 \mathrm{H}, \mathrm{NH}), 10.38$ (s, 1H, NH), $7.03-7.70$ (m, 23H, Ar-H); MS m/z (\%): 659 ( $\mathrm{M}^{+}$, 44.26), 597 (63.93), 476 (27.87), 433 (56.84), 366 (51.64), 328 (63.93), 296 (42.62), 73 (100); Anal. Calcd. for $\mathrm{C}_{38} \mathrm{H}_{31} \mathrm{ClN}_{4} \mathrm{OS}_{2}$ ( 659.26): C, 69.23; H, 4.74; Cl, 5.38; N, 8.50; S, 9.73. Found: C, 69.03; H, 4.54; Cl, 5.08; N, 8.30; S, 9.53.

\subsubsection{2-[1-(4-Chlorophenyl)-4,5-diphenyl-1H-imidazol-2-ylthio]-2-(3,4-diphenyl-thiazol- 2(3H)-ylidene)- $N$ - $p$-tolylacetamide 21 and 2-[1-(4-Chlorophenyl)-4,5-diphenyl-1H- imidazol-2-ylthio]-2-(4-oxo-3-phenyl-1,3-thiazolidin-2-ylidene)- $N$-p-tolylacetamide 22}

To a cold suspension of powdered potassium hydroxide $(0.01 \mathrm{~mol})$ in DMF $(20 \mathrm{ml})$ was added compound 2 
(0.01 mol) and phenyl isothiocyanate $(0.01 \mathrm{~mol})$. The reaction mixture was stirred at room temperature for $6 \mathrm{~h}$, and then treated with phenacyl bromide and/or ethyl chloroacetate $(0.01 \mathrm{~mol})$ and the stirring was continued at room temperature for further $10 \mathrm{~h}$. The reaction mixture was poured into $50 \mathrm{ml}$ of cold water. The result solid products were collected by filtration and recrystallized from a mixture of ethanol/DMF (1:1) to give compounds 21 and 22.

21: Yellow powder. Yield: 65\%. M.p.: $240^{\circ} \mathrm{C}-242^{\circ} \mathrm{C}$; IR ( $\left.\mathrm{KBr}, \mathrm{cm}^{-1}\right)$ : $3248(\mathrm{NH}), 1659(\mathrm{C}=\mathrm{O}), 3056,2937$,

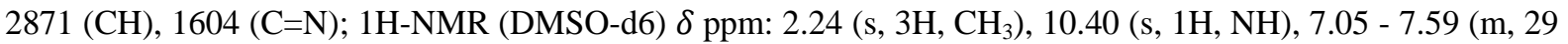
$\mathrm{H}, \mathrm{Ar}-\mathrm{H}$ and H-5 thiazoline); MS m/z (\%):745 (M+, 43.29), 695 (40.24), 577 (41.40), 450 (39.63), 421 (36.59), 313 (43.29), 217 (50.00), 172 (46.34), 111 (100). Anal. Calcd. for $\mathrm{C}_{45} \mathrm{H}_{33} \mathrm{Cl} \mathrm{N}_{4} \mathrm{OS}_{2}$ (745.35): C, 72.51; H, 4.46; Cl, 4.76; N, 7.52; S, 8.60. Found: C, 72.31; H, 4.26; Cl, 4.46; N, 7.32; S, 8.50.

22: Pale grey crystals. Yield: $62 \%$. M.p.: $228^{\circ} \mathrm{C}-230^{\circ} \mathrm{C}$; IR $\left(\mathrm{KBr}, \mathrm{cm}^{-1}\right): 3245(\mathrm{NH}), 3084,2929,2873(\mathrm{CH})$, 1726, 1657 (C=O); 1H-NMR (DMSO-d6) $\delta$ ppm: 2.25 (s, 3H, $\mathrm{CH}_{3}$ ), 10.41 (s, 1H, NH), $7.06-7.60$ (m, $23 \mathrm{H}$, Ar-H), 4.13 (s, 2H, CH ); MS m/z (\%): 670 ( $\left.\mathrm{M}^{+}-\mathrm{CH}_{3}, 12.66\right), 654$ (10.97), 594 (19.83), 503 (14.77), 475 (12.24), 348 (10.97), 139 (14.98), 125 (18.35), 55 (100). Anal. Calcd. for $\mathrm{C}_{39} \mathrm{H}_{29} \mathrm{ClN}_{4} \mathrm{O}_{2} \mathrm{~S}_{2}$ (685.25): C, 68.36; H, 4.27; Cl, 5.17; N, 8.18; S, 9.36. Found: C, 68.06; H, 4.07; Cl, 5.00; N, 8.00; S, 9.16.

\subsubsection{2-[5-Benzylidene-4-oxo-3-phenyl-1,3-thiazolidin-2-ylidene]-2-[1-(4-chlorophenyl)-4,5- diphenyl-1H-imidazol-2-ylthio]- $N$ - $p$-tolylacetamide 23}

To a well-stirred solution of compound $22(0.01 \mathrm{~mol})$ in DMF $(20 \mathrm{ml})$, piperidine $(0.2 \mathrm{ml})$ and benzaldehyde $(0.01 \mathrm{~mol})$ were added. The reaction mixture was stirred at $80^{\circ} \mathrm{C}$ for $3 \mathrm{~h}$. The separated crystals was filtered, dried and recrystallized from ethanol to give 23 as white crystals. Yield: $60 \%$. M.p.: $252^{\circ} \mathrm{C}-254^{\circ} \mathrm{C}$; IR $(\mathrm{KBr}$, $\mathrm{cm}^{-1}$ ): $3249(\mathrm{NH}), 3090,2997(\mathrm{CH}), 1657,1734$ (C=O); 1H-NMR (DMSO-d6) $\delta$ ppm: 2.25 (s, 3H, $\left.\mathrm{CH}_{3}\right), 10.53$ (s, 1H, NH), $7.03-7.70$ (m, 29H, Ar-H and =CH); MS m/z (\%):773 (M+, 6.72), 723 (77.3), 707 (57.14), 570 (50.42), 479 (54), 388 (100), 372 (58.82) Anal. Calcd. for $\mathrm{C}_{46} \mathrm{H}_{33} \mathrm{ClN}_{4} \mathrm{O}_{2} \mathrm{~S}_{2}$ (773.36): C, 71.44; H, 4.30; Cl, 4.58; N, 7.24; S, 8.29. Found: C, 71.24; H, 4.00; Cl, 4.28; N, 7.04; S, 8.09.

\subsection{Antimicrobial Assays}

Synthesized compounds 2, 3, 6, 13, 15a, 15b, 17, 20, 21, 22 and 23 were screened for their antimicrobial activities in vitro against two species of Gram-positive bacteria, namely Staphylococcus aureus (RCMB 0100010) and Bacillus subtilis (RCMB 010067), two Gram-negative bacteria, namely Pseudomonas aeuroginosa (RCMB 010043) and Escherichia coli (RCMB 010052) and against four species of fungi, namely Aspergillus fumigatus (RCMB 02568), Syncephalastrum racemosum (RCMB 05922), Geotrichum candidum (RCMB 05097) and Candida albicans (RCMB 05036). The antibacterial and antifungal activities were determined by means of inhibition $\% \pm$ standard deviation at a concentration of $100 \mu \mathrm{g} / \mathrm{ml}$ of tested samples [5]-[7]. Optical densities of antimicrobial were measured after 24 hours at $37^{\circ} \mathrm{C}$ to bacteria and measured after 48 hours at $28^{\circ} \mathrm{C}$ to fungal using a multidetection microplate reader at the Regional Center for Mycology and Biotechnology (Sun Rise-Tecan, USA at $600 \mathrm{~nm}$ ) Al-Azhar University. Ampicillin, gentamicin and amphotericin B were used as references to evaluate the potency of the tested compounds under the same conditions.

\section{Results and Discussion}

\subsection{Chemistry}

The synthetic procedures adopted to obtain the target compounds are depicted in Schemes 1-4. S-Alkylation of 1-(4-chlorophenyl)-4,5-diphenyl-1H-imiazole-2-thione 1 with 2-chloro- $N$-p-tolylacetamide afforded the corresponding 2-(1H-imidazol-2-ylthio)- $N$-p-tolylacetamide derivative 2 . The assignment of structure 2 was based on both elemental analysis and spectral data. 1H-NMR spectrum of 2 in (DMSO-d6) revealed signals at 2.24 ppm corresponding to $\mathrm{CH}_{3}$ group and a single at $4.11 \mathrm{ppm}$ for $\mathrm{CH}_{2}$ group. Moreover, mass spectrum showed a molecular ion peak at $\mathrm{m} / \mathrm{z} 510$ corresponding to a molecular formula $\mathrm{C}_{30} \mathrm{H}_{24} \mathrm{ClN}_{3} \mathrm{OS}$. Further evidence for the structure of compound 2 was obtained through studying their chemical reactivity via some chemical reactions. Thus, interaction of compound 2 with $p$-chlorobenzaldehyde yielded the arylidene derivative 3 (Scheme 1). 1H-NMR spectrum in (DMSO-d6) of $\mathbf{3}$ show the disappearance of $\mathrm{CH}_{2}$ protons observed with the respective starting precursors 2 at $\delta 4.11 \mathrm{ppm}$, and the appearance multiple signals in the region at $\delta 6.41-7.27$ ppm corresponding to 


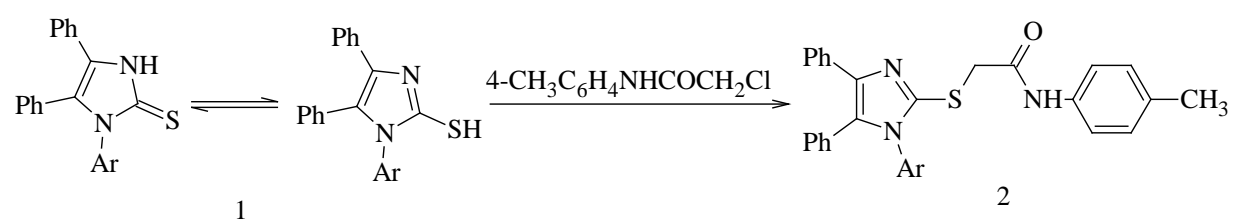

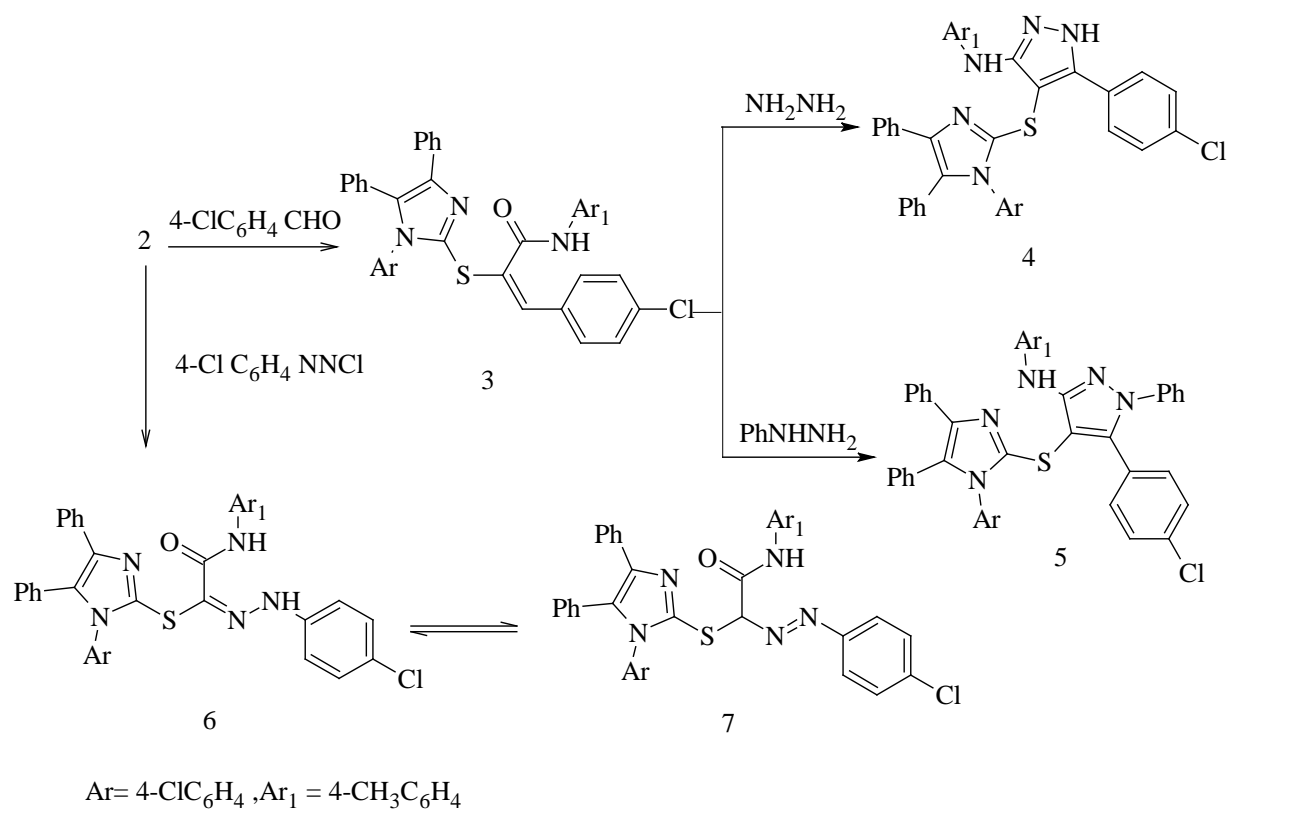

Scheme 1. Formation of compounds 2-6.

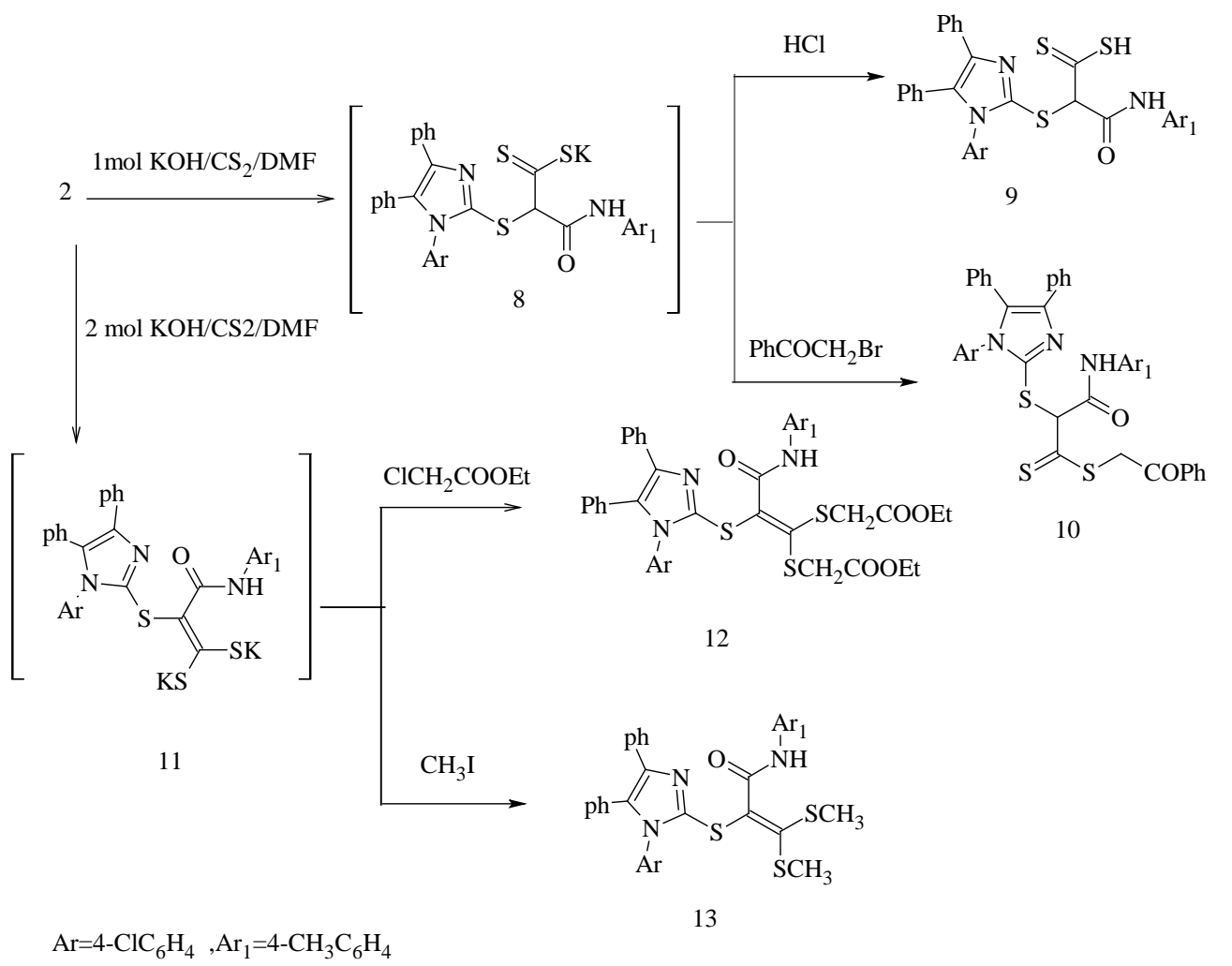

Scheme 2. Formation of compounds 8-13. 


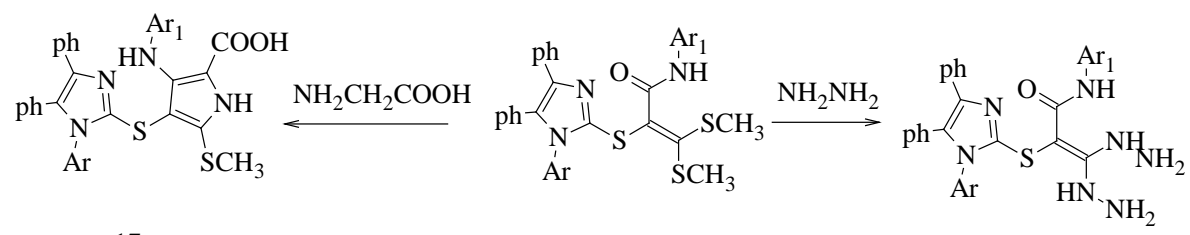

17

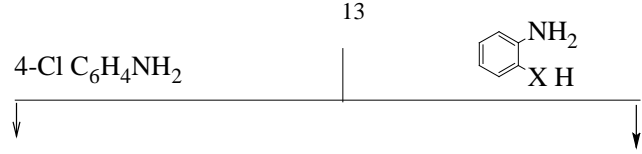

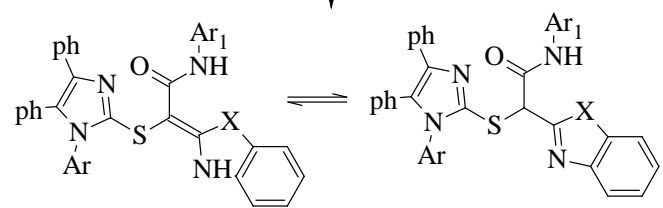

15

$\mathrm{Ar}=\mathrm{p}-\mathrm{ClC}_{6} \mathrm{H}_{4}, \mathrm{Ar}_{1}=\mathrm{p}-\mathrm{CH}_{3} \mathrm{C}_{6} \mathrm{H}_{4}$

$\mathrm{a}, \mathrm{X}=\mathrm{NH}$

$\mathrm{b}, \mathrm{X}=\mathrm{O}$

Scheme 3. Formation of compounds 14-17.<smiles>[14CH3]NC(=O)/C(Sc1nc(-c2ccccc2)c(-c2ccccc2)n1[Al])=C1\SCC(=O)N1c1ccccc1</smiles><smiles>CC(C)NC(=O)/C(Sc1nc(-c2ccccc2)c(-c2ccccc2)n1[Al])=c1\s/c(=C\c2ccccc2)c(=O)n1-c1ccccc1</smiles>

23

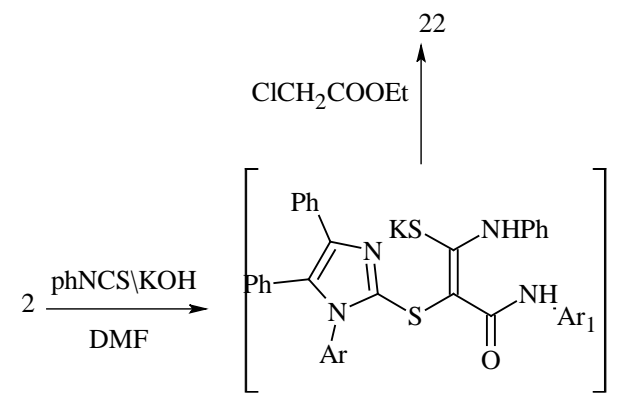

18<smiles>C=CCn1c(SC(C(=O)NCC(C)C)C(=S)Nc2ccccc2)nc(-c2ccccc2)c1-c1ccccc1</smiles>

A<smiles>CC(C)NC(=O)/C(Sc1nc(-c2ccccc2)c(-c2ccccc2)n1[Al])=C(/S)Nc1ccccc1</smiles>

B

19

$\mathrm{Ar}=\mathrm{p}-\mathrm{ClC}_{6} \mathrm{H}_{4}, \mathrm{Ar}_{1}=\mathrm{p}-\mathrm{CH}_{3} \mathrm{C}_{6} \mathrm{H}_{4}$

Scheme 4. Formation of compounds 18-22.

the aromatic protons together with $1 \mathrm{H}$ benzylidene $\mathrm{C}=\mathrm{CH}$ proton. Mass spectrum of $\mathbf{3}$ showed a molecular ion peak at $\mathrm{m} / \mathrm{z} 632$ corresponding to the molecular formula $\mathrm{C}_{37} \mathrm{H}_{27} \mathrm{Cl}_{2} \mathrm{~N}_{3} \mathrm{OS}$ (see experimental section).

Reaction of arylidene derivatives 3 with hydrazine hydrate in ethanol [8] and few drop of acetic acid gave the 
corresponding $1 H$-pyrazol-3-amine derivative 4 . While, reaction 3 with phenyl hydrazine in the presence of acetic acid and few drops of hydrochloric acid [9] afforded the corresponding 1-phenyl-1H-pyrazol-3-amine $\mathbf{5}$. The structure of the pyrazole derivatives $\mathbf{4}$ and $\mathbf{5}$ were established on the basis of analytical and spectral data. The IR spectrum of $\mathbf{4}$ showed the disappearance of absorption band of $\mathrm{C}=\mathrm{O}$ group and appearance of new absorption band of $\mathrm{NH}$ at $3238 \mathrm{~cm}^{-1}$. Mass spectrum of 4 showed a molecular ion peak at $\mathrm{m} / \mathrm{z} 644$ corresponding to the molecular formula $\mathrm{C}_{37} \mathrm{H}_{27} \mathrm{Cl}_{2} \mathrm{~N}_{5} \mathrm{~S}$.

Diazotization of $p$-chloroaniline followed by coupling with active methylene group in compound $\mathbf{2}$ in pyridine yielded the hydrazone form 6 rather than the azo form $\mathbf{7}$ based on spectral data [10]. The 1H-NMR spectrum of compound 6 recorded in (DMSO-d6) revealed a signal at $\delta 8.58 \mathrm{ppm}$, which could be attributed to hydrazone NH group (Scheme 1).

Reaction of compound 2 with carbon disulphide and one equivalent potassium hydroxide in dimethylformamide (DMF) gave non-isolated intermediate potassium salt $\mathbf{8}$. Treatment of the non-isolable potassium salts $\mathbf{8}$ with dilute hydrochloric acid [11] afforded the corresponding 2-(1H-imidazol-2-ylthio)-2-(p-tolylcarbamoyl) ethanedithioic acid $\mathbf{9}$. The assignment of structure 9 was based on both elemental analysis and spectral data. The IR spectrum displayed absorptions band at $3231 \mathrm{~cm}^{-1}(\mathrm{NH})$ and $1271 \mathrm{~cm}^{-1}(\mathrm{C}=\mathrm{S})$. The mass spectrum showed the molecular ion peak at $\mathrm{m} / \mathrm{z} 586$ corresponding to the molecular formula $\mathrm{C}_{31} \mathrm{H}_{24} \mathrm{ClN}_{3} \mathrm{OS}_{3}$. On the other hand, treatment of intermediate salt 8 with phenacyl bromide [12] to give the corresponding 3-[benzoylmethylthio]-2-[1 $H$-imidazol-2-ylthio]- $N$ - $p$-tolyl-3-thioxo-propaneamide derivatives $\mathbf{1 0}$. The $1 \mathrm{H}$-NMR spectra of $\mathbf{1 0}$ in (DMSO-d6) revealed a single at $\delta 4.39 \mathrm{ppm}$ and $\delta 4.57 \mathrm{ppm}$ assigned to the $\mathrm{CH}_{2}$ and $\mathrm{CH}$ protons respectively (Scheme 2).

While the reaction 2 with carbon disulphide in the presence of two equivalent potassium hydroxide in DMF to give non-isolated potassium salt 11, which was allowed to react with halogenated compounds namely ethylchloroacetate [13] and methyl iodide [14] afforded the corresponding 3,3-bis[(ethoxycarbonyl)methylthio]- $N$ - $p$-tolylacrylamide 12 and 3,3-bis-(methylthio)- $N$-p-tolylacrylamide 13 respectively. We suggest a mechanism for the formation of $\mathbf{1 2}$ in which the intermediate $\mathbf{I}$ is obtained first, then elimination of potassium chloride (Figure $\mathbf{1}$ ).

The structure of synthesis compound $\mathbf{1 2}$ and $\mathbf{1 3}$ ware elucidated on the basis of the elemental analysis and spectral data. For example, 1H-NMR spectra in (DMSO-d6) of 12 displayed two multiple at 1.13 - 1.23 and 4.04 4.13 for ethoxy protons of two carboethoxy group and two single at 4.33 and $4.37 \mathrm{ppm}$ for two methylene protons. On the other hand, $1 \mathrm{H}-\mathrm{NMR}$ spectrum (DMSO-d6) of $\mathbf{1 3}$ showed single signal at $\delta 2.28 \mathrm{ppm}$ for 6 protons of two similar methyl protons. The mass spectrum of compound 13 showed a molecular ion peak at $\mathrm{m} / \mathrm{z} 614$ corresponding to a molecular formula $\mathrm{C}_{33} \mathrm{H}_{28} \mathrm{ClN}_{3} \mathrm{OS}_{3}$ (Scheme 2).

Moreover, condensation of $\mathbf{1 3}$ with hydrazine hydrate afforded the corresponding 3, 3-dihydrazino- $N$ - $p$-tolylacrylamide derivative $\mathbf{1 4}$. The structure of $\mathbf{1 4}$ was identified as the reaction product on the basis of its elemental analysis and spectral data. The 1H-NMR spectrum of $\mathbf{1 4}$ showed a multiple signals in the region at $\delta 7.09-7.56$ ppm corresponding to the aromatic protons together with the NH proton, two single signals at $\delta 4.84 \mathrm{ppm}$ and 4.89 corresponding to the two $\mathrm{NH}_{2}$ protons, and another two single signals at $\delta 10.33$ and $\delta 10.51 \mathrm{ppm}$ assignable to two NH protons. Mass spectrum of $\mathbf{1 4}$ showed a molecular ion peak at $\mathrm{m} / \mathrm{z} 582$ corresponding to a molecular formula $\mathrm{C}_{31} \mathrm{H}_{28} \mathrm{ClN}_{7} \mathrm{OS}$. In addition, the condensation of $\mathbf{1 3}$ with suitable amine namely o-phenylenediamine, and $o$-aminophenol [15] in refluxing absolute ethanol to afford the corresponding 2-(1H-benzimidazol2-yl)- and 2-(1,3-benzoxazol-2-yl)- $N$-p-tolylacetamide 15a, b respectively (Scheme 3$)$. The structures of compounds 15a, b were established and confirmed by their elemental analysis and spectral data (see experimental section). The formation of $\mathbf{1 5} \mathbf{~ a , ~ b}$ were assumed to proceed through nucleophilic attack of the two -NH2 group in $o$-phenylenediamine,or $\mathrm{NH}, \mathrm{OH}$ groups in $o$-aminophenol to the ethylenic double bond in the compound $\mathbf{1 3}$ followed by elimination of two moles of methyl mercaptan (Figure 2).

$S, S$-acetals 13 was converted to corresponding $S, N$-acetals by reacting with appropriate primary. Thus, reaction of 13 with p-chloroaniline afforded ketene $N, S$-acetals 16 (Scheme 3). The assignment of the structure of 16 was based on spectral data. The IR spectrum of $\mathbf{1 6}$ showed absorption bands at 3246, $3191 \mathrm{~cm}^{-1}$ for two NH. Its 1H-NMR spectrum (DMSO-d6) of $\mathbf{1 6}$ showed single signal at $\delta 2.39 \mathrm{ppm}$ for $\mathrm{SCH}_{3}$ protons, $\delta 7.09-7.62 \mathrm{ppm}$ corresponding to the aromatic protons together with the NH proton and single signals at $\delta 10.29 \mathrm{ppm}$ assignable to $\mathrm{NH}$. The mass spectrum of $\mathbf{1 6}$ showed a molecular ion peak at $\mathrm{m} / \mathrm{z} 693$ corresponding to the molecular formula $\mathrm{C}_{38} \mathrm{H}_{30} \mathrm{Cl}_{2} \mathrm{~N}_{4} \mathrm{OS}_{2}$.

Furthermore, the reaction of $\mathbf{1 3}$ with Glycine in ethanol containing triethylamine [16] afforded the corresponding $1 H$-pyrrole-2-carboxylic acid derivatives 17 (Scheme 3). The assignment of the structure of $\mathbf{1 7}$ was 


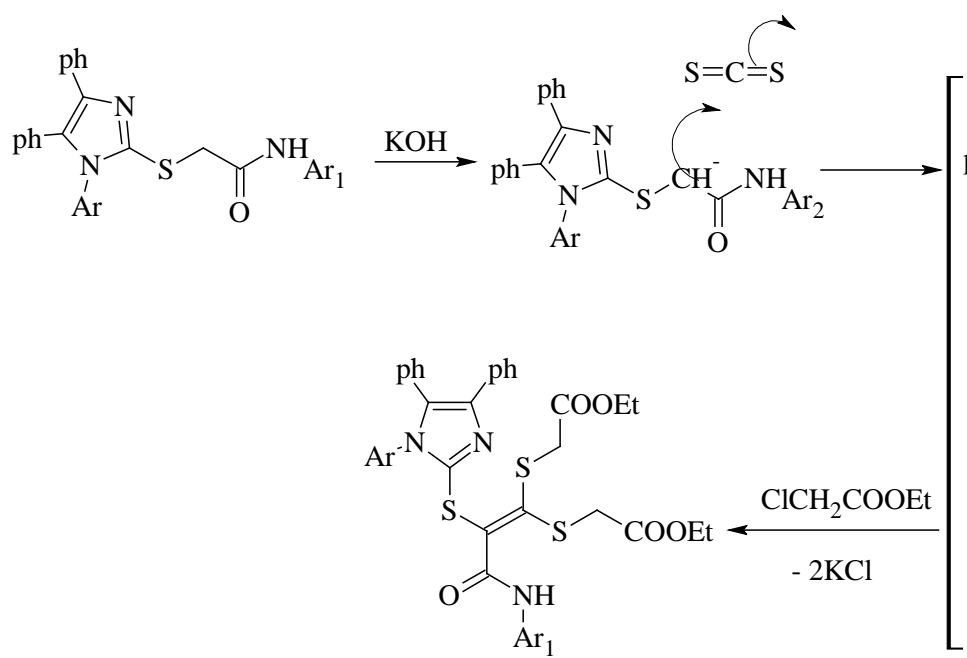

12

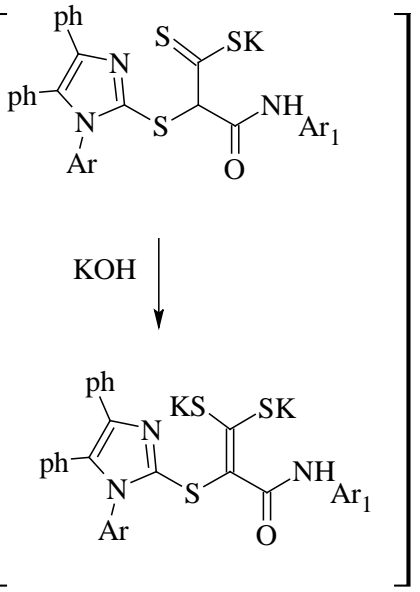

I

Figure 1. Proposed mechanism formation of 3,3-bis[(ethoxycarbonyl)methylthio]- $N$ - $p$-tolylacrylamide derivatives 12 .

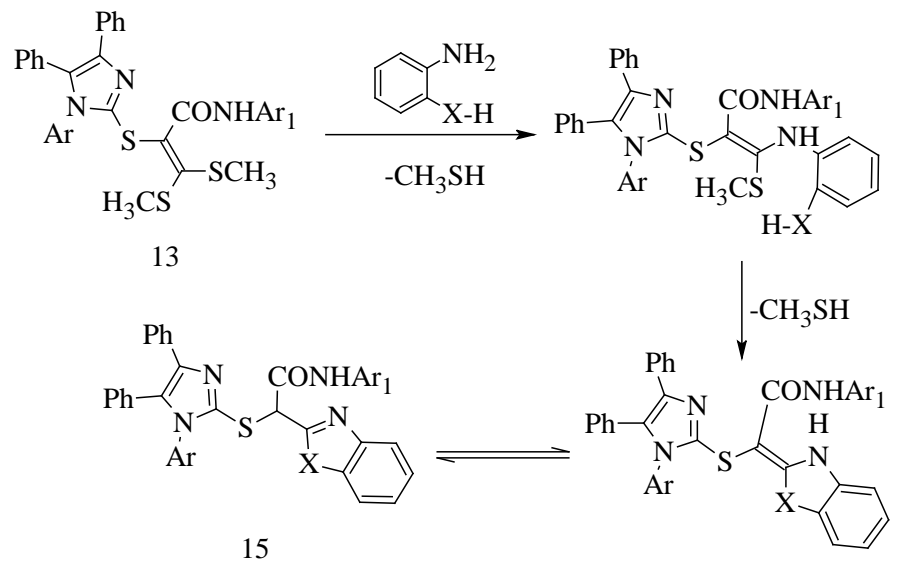

Figure 2. The proposed mechanism formation 15a, b.

based on spectral data. The IR spectrum of 17 showed absorption bands at $3436 \mathrm{~cm}^{-1}(\mathrm{OH})$ and $3247,3184 \mathrm{~cm}^{-1}$ (NH). Its 1H-NMR spectrum (DMSO-d6) single signal at $\delta 2.29 \mathrm{ppm}$ for $\mathrm{SCH}_{3}$ protons, $\delta 7.09-7.47 \mathrm{ppm}$ corresponding to the aromatic protons together with the $2 \mathrm{NH}$ protons. The mass spectrum of $\mathbf{1 7}$ showed a molecular ion peak at $m / z 623$ corresponding to the molecular formula $\mathrm{C}_{34} \mathrm{H}_{27} \mathrm{Cl} \mathrm{N}_{4} \mathrm{O}_{2} \mathrm{~S}_{2}$.

2-(1H-imidazol-2-ylthio)- $N$ - $p$-tolylacetamide 2 was utilized as a key intermediate for the synthesis of thiocarbamoyl derivative 19 via its reaction with phenyl isothiocyanate. Thus, reaction $\mathbf{2}$ with phenyl isothiocyanate in DMF in the presence of an equimolar amount of potassium hydroxide yielded the non-isolable intermediate potassium sulphide salt 18. Acidification of the potassium salt $\mathbf{1 8}$ with dilute hydrochloric acid afforded the corresponding thiocarbamoyl derivative 19, which can exist in two tautomeric thione-thiol forms A and B (Scheme 4). Assignment of the product 19 was based on elemental analysis and spectral data. 1H-NMR spectrum displayed multiple signals at $\delta 7.05-7.59 \mathrm{ppm}$ for aromatic protons and two single signals at $\delta 10.40$ and $10.20 \mathrm{ppm}$ assignable to two NH protons. Mass spectrum showed a molecular ion peak at $\mathrm{m} / \mathrm{z} 645$ corresponding to a molecular formula $\mathrm{C}_{37} \mathrm{H}_{29} \mathrm{ClN}_{4} \mathrm{OS}_{2}$.

Treatment of the non-isolable potassium sulfide salt $\mathbf{1 8}$ with methyl iodide [17] afforded the ketene N, Sacetal 20. The structure of $\mathbf{2 0}$ was established on the basis of its elemental analysis and spectral data. Its IR spectrum showed absorption bands at 3246, $3191 \mathrm{~cm}^{-1}$ due to two NH groups. On addition $1 \mathrm{H}$ NMR spectrum (DMSO-d6) displayed single signal at $\delta 2.54 \mathrm{ppm}$ for $\mathrm{SCH}_{3}$. The mass spectrum showed a molecular ion peak at 
$\mathrm{m} / \mathrm{z} 659$ corresponding to a molecular formula $\mathrm{C}_{38} \mathrm{H}_{31} \mathrm{ClN}_{4} \mathrm{OS}_{2}$ (Scheme 4).

On the other hand, reaction of $\mathbf{1 8}$ with phenacyl bromide and ethyl chloroacetate [18] afforded 2-(3, 4-diphenyl-1,3-thiazol-2(3H)-ylidene)- and 2-(4-oxo-3-phenyl-1,3-thiazolidin-2-ylidene)- $N$-p-tolylacetamide 21 and $\mathbf{2 2}$ respectively. The structures of compounds $\mathbf{2 1}$ and $\mathbf{2 2}$ were established and confirmed by their elemental analysis and spectral data. The 1H-NMR spectrum of 21 showed a multiple signals in the region at $\delta 7.05-7.59$ ppm corresponding to the aromatic protons together with the H-5 protons of the thiazole ring and a single signal at $\delta 10.40 \mathrm{ppm}$ for $\mathrm{NH}$ proton. Mass spectrum of 21 revealed a molecular ion peak at $\mathrm{m} / \mathrm{z} 745$ corresponding to a molecular formula $\mathrm{C}_{45} \mathrm{H}_{33} \mathrm{ClN}_{4} \mathrm{OS}_{2}$. The IR spectrum of 22 showed absorption bands at $1726 \mathrm{~cm}^{-1}$ due to $\mathrm{CO}$ of thiazolidinone ring. The $1 \mathrm{H}-\mathrm{NMR}$ spectrum of 22 showed a single signal equivalent to two protons at $\delta 4.13$ ppm which represent the $\mathrm{CH}_{2}$ protons of the thiazolidinone ring. The Claisene Schmidt condensation of thiazolidin-5-one 22 with benzaldehyde [19] in DMF and in the presence of a catalytic amount of piperidine afforded arylidene derivatives 23 (Scheme 4). The structures of latter products were confirmed based on elemental analysis and spectral data (see experimental section).

\subsection{Antimicrobial Activity}

Synthesized compounds 2, 3, 6, 13, 15a, 15b, 17, 20, 21, 22 and 23 were evaluated for antibacterial and antifungal activities.

\subsubsection{Antibacterial Activity}

Synthesized compounds 2, 3, 6, 13, 15a, 15b, 17, 20, 21, 22 and 23 were screened for their antibacterial activities in vitro against Gram-positive namely Staphylococcus aureus (RCMB 0100010) and Bacillus subtilis (RCMB 010067) and Gram- negative Pseudomonas aeuroginosa (RCMB 010043) and Escherichia coli (RCMB 010052). Ampicillin and gentamicin were used as references to evaluate the potency of the tested compounds. The inhibitory effects of the synthetic compounds against these organisms are given in Table 1, Figure 3.

In general, most of the tested compounds revealed better activity against the Gram-positive bacteria rather than the Gram-negative bacteria. Compounds 3, 13 and 15a exhibited excellent antibacterial activity against the tested organisms while compounds 15b, 17, 20, 21 and 23 showed moderate antibacterial activity against the tested organisms and compound $\mathbf{2 , 6}$ and $\mathbf{2 2}$ showed weak antibacterial activity against the tested organisms. In addition, all test compounds were found to be inactive against Pseudomonas aeuroginosa (RCMB 010043).

\subsubsection{Antifungal Activity}

The newly synthesized compounds 2, 3, 6, 13, 15a, 15b, 17, 20, 21, 22 and 23 were screened for their antifungal activities in vitro against, Aspergillus fumigatus (RCMB 02568), Syncephalastrum racemosum (RCMB 05922), Geotrichum candidum (RCMB 05097) and Candida albicans (RCMB 05036). Amphotericin B was used as standards to evaluate the potency of the tested compounds. The inhibitory effects of the synthetic compounds against these organisms are given in Table 2, Figure 4.

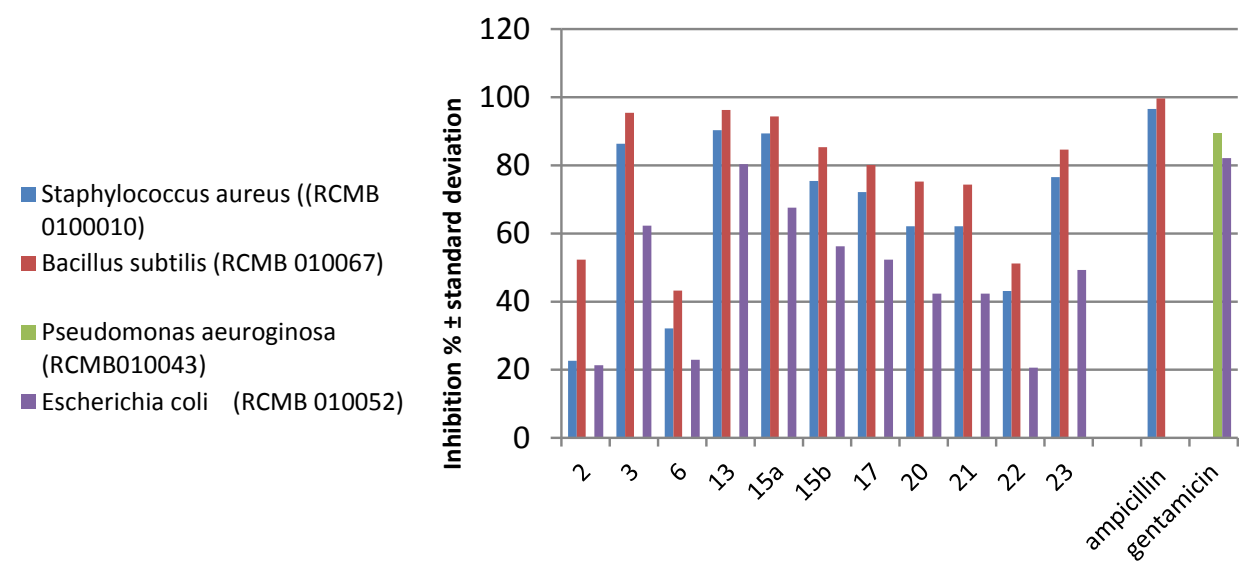

Figure 3. Graphical representation of the antibacterial activity of tested compounds compared to ampicillin and gentamicin. 


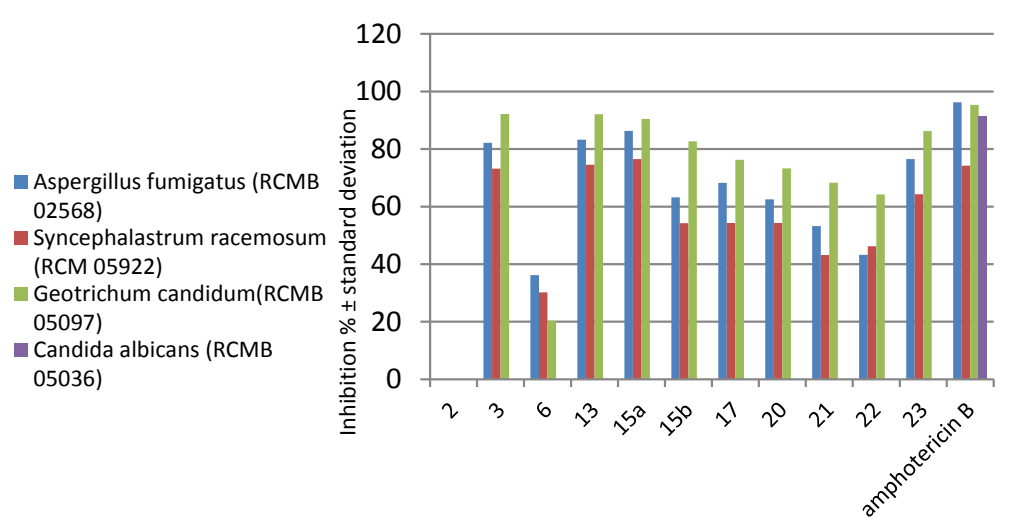

Figure 4. Graphical representation of the antifungal activity of tested compounds compared to amphotericin B.

Table 1. Antibacterial evaluation of the some synthesized compounds.

\begin{tabular}{|c|c|c|c|c|}
\hline \multirow{3}{*}{ Comp. No. } & \multicolumn{4}{|c|}{ Inhibition $\% \pm$ standard deviation } \\
\hline & \multicolumn{2}{|c|}{ Gram-positive bacteria } & \multicolumn{2}{|c|}{ Gram-negative bacteria } \\
\hline & $\begin{array}{l}\text { Staphylococcus aureus } \\
\text { ((RCMB 0100010) }\end{array}$ & $\begin{array}{l}\text { Bacillus subtilis } \\
\text { (RCMB 010067) }\end{array}$ & $\begin{array}{l}\text { Pseudomonas aeuroginosa } \\
\text { (RCMB 010043) }\end{array}$ & $\begin{array}{l}\text { Escherichia coli } \\
\text { (RCMB 010052) }\end{array}$ \\
\hline 2 & $22.63 \pm 0.25$ & $52.31 \pm 0.37$ & NA & $21.36 \pm 0.44$ \\
\hline 3 & $86.32 \pm 0.44$ & $95.41 \pm 0.25$ & NA & $62.31 \pm 0.25$ \\
\hline 6 & $32.12 \pm 0.63$ & $43.25 \pm 0.42$ & NA & $22.96 \pm 0.16$ \\
\hline 13 & $90.31 \pm 0.43$ & $96.25 \pm 0.53$ & NA & $80.36 \pm 0.33$ \\
\hline $15 a$ & $89.35 \pm 0.15$ & $94.32 \pm 0.42$ & NA & $67.58 \pm 0.53$ \\
\hline $15 b$ & $75.44 \pm 0.44$ & $85.34 \pm 0.58$ & NA & $56.23 \pm 0.19$ \\
\hline 17 & $72.13 \pm 0.44$ & $80.12 \pm 0.63$ & NA & $52.34 \pm 0.25$ \\
\hline 20 & $62.14 \pm 0.44$ & $75.24 \pm 0.63$ & NA & $42.36 \pm 0.25$ \\
\hline 21 & $62.14 \pm 043$ & $74.32 \pm 0.53$ & NA & $42.32 \pm 0.25$ \\
\hline 22 & $43.12 \pm 0.44$ & $51.21 \pm 0.25$ & NA & $20.63 \pm 0.33$ \\
\hline 23 & $76.52 \pm 0.2$ & $84.63 \pm 0.3$ & NA & $49.32 \pm 0.3$ \\
\hline \multicolumn{3}{|c|}{ Reference drugs } & & \\
\hline Ampicillin & $96.52 \pm 0.2$ & $99.65 \pm 0.3$ & & \\
\hline Gentamicin & & & $89.23 \pm 0.1$ & $82.14 \pm 0.3$ \\
\hline
\end{tabular}

Table 2. Antifungal evaluation of the some synthesized compounds.

\begin{tabular}{|c|c|c|c|c|}
\hline \multirow[b]{2}{*}{ Comp. No. } & \multicolumn{4}{|c|}{ Inhibition $\% \pm$ standard deviation } \\
\hline & $\begin{array}{l}\text { Aspergillus fumigatus } \\
\text { (RCMB 02568) }\end{array}$ & $\begin{array}{c}\text { Syncephalastrum racemosum } \\
\text { (RCMB 05922) }\end{array}$ & $\begin{array}{l}\text { Geotrichum candidum } \\
\text { (RCMB 05097) }\end{array}$ & $\begin{array}{l}\text { Candida albicans } \\
\text { (RCMB 05036) }\end{array}$ \\
\hline 2 & NA & NA & NA & NA \\
\hline 3 & $82.21 \pm 0.53$ & $73.21 \pm 0.44$ & $92.14 \pm 0.58$ & NA \\
\hline 6 & $36.21 \pm 0.44$ & $30.21 \pm 0.37$ & $20.36 \pm 0.25$ & NA \\
\hline 13 & $83.25 \pm 0.25$ & $74.52 \pm 0.25$ & $92.13 \pm 0.38$ & NA \\
\hline $15 a$ & $86.32 \pm 0.33$ & $76.52 \pm 0.25$ & $90.43 \pm 0.34$ & NA \\
\hline $15 b$ & $63.22 \pm 0.34$ & $54.25 \pm 0.25$ & $82.68 \pm 0.58$ & NA \\
\hline 17 & $68.23 \pm 0.39$ & $54.32 \pm 0.58$ & $76.24 \pm 0.58$ & NA \\
\hline 20 & $62.52 \pm 0.39$ & $54.32 \pm 0.16$ & $73.25 \pm 0.58$ & NA \\
\hline 21 & $53.21 \pm 0.58$ & $43.20 \pm 25$ & $68.30 \pm 0.35$ & NA \\
\hline 22 & $43.25 \pm 0.25$ & $46.24 \pm 0.58$ & $64.25 \pm 0.17$ & NA \\
\hline 23 & $76.52 \pm 0.25$ & $64.31 \pm 0.44$ & $86.23 \pm 0.63$ & NA \\
\hline Amphotericin B & $96.25 \pm 0.1$ & $74.25 \pm 0.2$ & $95.36 \pm 0.2$ & $91.29 \pm 0.1$ \\
\hline
\end{tabular}


Compounds 13, 15a exhibited excellent antifunger activity, which is better than the amphotericin B against Syncephalastrum racemosum (RCMB 05922), while its strong antifunger activity against Aspergillus fumigatus (RCMB 02568) and Geotrichum candidum (RCMB 05097) is comparable to amphotericin B. The compounds 3 , 15b, 17, 20, 21, 22 and 23 showed strong moderate activity against Aspergillus fumigatus (RCMB 02568), Syncephalastrum racemosum (RCMB 05922) and Geotrichum candidum (RCMB 05097) compared to amphotericin B against. While the compounds 6 weak antifungal activity against Aspergillus fumigatus (RCMB 02568), Syncephalastrum racemosum (RCMB 05922), Geotrichum candidum (RCMB 05097). On the other hand, compound 2 inactive against all organism. Furthermore, all test compounds were found to be inactive against Candida albicans (RCMB 05036).

\section{Conclusion}

In this paper, we report the synthesis of 2-(1H-imidazol-2-ylthio)- $N$-p-tolylacetamide 2 . The active methylene moiety of compound 2 was allowed to react with $\mathrm{CS}_{2}$ and/or phenyl isothiocyanate in dimethylformamide in the presence of potassium hydroxide and yielded the non-isolable intermediate potassium sulphide salt 8, 11 and 18, which is used as intermediate to synthesis series of novel substituted imidazole derivatives in good yield. Synthesized compounds 2, 3, 6, 13, 15a, 15b, 17, 20, 21, 22 and 23 were evaluated for antibacterial and antifungal activities. Most of the tested compounds revealed better activity against the Gram-positive rather than the Gramnegative bacteria. Compound 13 exhibited excellent antibacterial activity against Staphylococcus aureus (RCMB 0100010), Bacillus subtilis (RCMB 010067) and Escherichia coli (RCMB 010052). Compounds 13, 15a exhibited excellent antifunger activity, which is better than the amphotericin B against Syncephalastrum racemosum (RCMB 05922).

\section{References}

[1] Dieter, R.K. (1986) $\alpha$-Oxo Ketene Dithioacetals and Related Compounds: Versatile Three-Carbon Synthons. Tetrahedron, 42, 3029-3096. http://dx.doi.org/10.1016/S0040-4020(01)87376-2

[2] Junjappa, H., Ila, H. and Asokan, C.V. (1990) $\alpha$-Oxoketene-S,S-, N,S- and N,N-acetals: Versatile Intermediates in Organic Synthesis. Tetrahedron, 46, 5423-5506. http://dx.doi.org/10.1016/S0040-4020(01)87748-6

[3] Khali, M.A., Sayed, S.M. and Raslan, M.A. (2012) Reactivity of 2-Cyano- $N$-(4-(1-methyl-1H-benzo[d]imidazol-2yl)-3-(methylthio)-1-phenyl-1H-pyrazol-5-yl)acetamide: A Facile Synthesis of Pyrazole, Thiazole, 1,3,4-Thiadiazole and Polysubstituted Thiophene Derivatives. American Journal of Organic Chemistry, 2, 161-170. http://dx.doi.org/10.5923/j.ajoc.20120206.06

[4] Abdelhamid, A.O and Afifi, M.A. (2010) Synthesis of Some New Thiazoles and Pyrazolo[1,5-a]pyrimidines Containing an Antipyrine Moiety. Synthetic Communications, 40, 1539-1550. http://dx.doi.org/10.1080/00397910903100726

[5] Kaya, E.G., Ozbilge, H. and Albayrak, S. (2009) Determination of Effect of Gentamicin against Staphylococcus aureus by Using Microbroth Kinetic System. Ankem Derg, 23, 110-114.

[6] Sofy, A.R., Hmed, A.A., Sharaf, A.M. and El-Dougdoug, K.A. (2014) Structural Changes of Pathogenic Multiple Drug Resistance Bacteria Treated with T. vulgaris Aqueous Extract. Nature and Science, 12, 83-88.

[7] Caldeira, E.M., Osório, A., Oberosler, E.L., Vaitsman, D.S., Alviano, D.S. and Nojima, M.D.G. (2013) Antimicrobial and Fluoride Release Capacity of Orthodontic Bonding Materials. Journal of Applied Oral Science, 21, 327-334. http://dx.doi.org/10.1590/1678-775720130010

[8] Shabaan, M., Taher, A. and Osman, E.O. (2011) Synthesis of Novel 3,4-Dihydroquinoxalin-2(1H)-one Derivatives. European Journal of Chemistry, 3, 365-371.

[9] Voskiene, A., Mickevicius, V. and Mikulskiene, G. (2007) Synthesis and Structural Characerization of Products Condensation 4-Carboxy-1-(4-styrylcarbonylphenyl)-2-pyrrolidinones with Hydrazines. ARKIVOC: Archive for Organic Chemistry, 2007, 303-314.http://dx.doi.org/10.3998/ark.5550190.0008.f29

[10] Mohare, R.M., Fleit, D.H. and Sakka, O.K. (2011) Novel Synthesis of Hydrazide-Hydrazone Derivatives and Their Utilization in the Synthesis of Coumarin, Pyridine, Thiazole and Thiophene Derivatives with Antitumor Activity. Molecules, 16, 16-27. http://dx.doi.org/10.3390/molecules16010016

[11] El-Bayouki, K.A., Basyounia, W.M., Mohamed, Y.A., Aly, M.M. and Abbas, S.Y. (2011) Novel 4(3H)-Quinazolinones Containing Biologically Active Thiazole, Pyridinone and Chromene of Expected Antitumor and Antifungal Activities. European Journal of Chemistry, 2, 455-462. http://dx.doi.org/10.5155/eurjchem.2.4.455-462.171

[12] Elgemeie, G.H., Elghandour, A.H., Ali, H.A. and Hussein, A.M. (2002) Novel 2-Thioxohydantoin Ketene Dithioacetals: 
Versatile Intermediates for Synthesis of Methylsulfanylimidazo[4,5-c]pyrazole and Methylsulfanylpyrrolo[1, 2-c]imidazoles. Synthetic Communications, 32, 2245-2253. http://dx.doi.org/10.1081/SCC-120005435

[13] Wang, Y., Dong, D., Yang, Y., Huang, J., Ouyang, Y. and Liu, Q. (2007) A Facile and Convenient One-Pot Synthesis of Polysubstituted Thiophenes from 1, 3-Dicarbonyl Compounds in Water. Tetrahedron, 63, 2724-2728. http://dx.doi.org/10.1016/j.tet.2006.12.090

[14] Khalil, A.M., Berghot, M.A. and Goudal, M.A. (2009) Synthesis and Antibacterial Activity of Some New Heterocycles Incorporating Phthalazine. European Journal of Medicinal Chemistry, 44, 4448-4454. http://dx.doi.org/10.1016/j.ejmech.2009.06.003

[15] Elgemeie, G.H., Elghandour, A.H. and Abd Elaziz, G.W. (2003) Novel Synthesis of Heterocyclic Ketene N,N-, N,O-, and N,S-Acetals Using Cyanoketene Dithioacetals. Synthetic Communications, 33, 1659-1664. http://dx.doi.org/10.1081/SCC-120018927

[16] Sommena, G.L., Comelb, A. and Kirsch, G. (2005) An Easy Access to Variously Substituted Pyrroles Starting from Ketene Dithioacetals. Synthetic Communications, 35, 693-699. http://dx.doi.org/10.1081/SCC-200050365

[17] Bondock, S., Rabie, R., Etman, H.A. and Fadda, A. (2008) Synthesis and Antimicrobial Activity of Some New Heterocycles Incorporating Antipyrine Moiety. European Journal of Medicinal Chemistry, 43, 2122-2129. http://dx.doi.org/10.1016/j.ejmech.2007.12.009

[18] Bondock, S., Fadaly, W. and Metwally, M.A. (2010) Synthesis and Antimicrobial Activity of Some New Thiazole, Thiophene and Pyrazole Derivatives Containing Benzothiazole Moiety. European Journal of Medicinal Chemistry, 45, 3692-3701. http://dx.doi.org/10.1016/j.ejmech.2010.05.018

[19] Rao, R.M., Reddy, G.N. and Sreeramulu, J. (2011) Synthesis of Some New Pyrazolo-Pyrazole Derivatives Containing Indoles with Antimicrobial Activity. Der Pharma Chemica, 3, 301-309. 